\title{
The Ensuring of Survivability of Wave Energy Stations Constructive Elements in Extreme Weather Conditions
}

\author{
Alexander Solovyev ${ }^{1}$, Dmitriy Solovyev ${ }^{2}$ and Liubov Shilova ${ }^{3, *}$ \\ ${ }^{1}$ Lomonosov Moscow State University, Department of Geography, 119991, GSP-1 Moscow, \\ Leninskie Gory, 1, Building 19 \\ ${ }^{2}$ Shirshov Institute of Oceanology, Russian Academy of Sciences, 117997, Moscow, Nakhimovsky \\ Prospect, 36 \\ ${ }^{3}$ Moscow State University of Civil Engineering, Yaroslavskoe shosse, 26, Moscow, 129337, Russia
}

\begin{abstract}
Current problems and the tasks of increasing the reliability of the equipment designs for utilizing the energy of sea waves are described in the article. The requirements for the protection of float and buoy structures from storm impacts by immersing them in undisturbed water layers using rotary engines articulated with a wave power station are formulated. Ways to protect wave converters from storm impacts are proposed.
\end{abstract}

\section{Introduction}

Since profitable differs of wave energy from other energy sources are discovered, energywave converters are of interest to specialists. The creation of wave energy systems required the fundamental research works about wave processes in the ocean, coastal zone and shelf. In each case, the use of wave energy is associated with the area of their utilization and the characteristics of the systems, that fact explains many patented design solutions in this area. Before 1980, about 1000 different various systems and design for the use of wave energy were recorded in 20 countries of the world [1,2].

The energy of the waves is characterized by their height. Energy exists in two forms: potential and kinetic (energy of motion). It is known that they are quantitatively equal and, therefore, the amplitude of the wave is an indicator of all energy. Another important characteristic is the frequency of wave oscillations, which depend on the acceleration of the wind - the distance in a straight line on which the wind runs from coast to this point. Knowing the acceleration and wind speed, the amplitude of the wave can be calculated. The period of sea waves can vary from two to twenty seconds. Waves can form a local wind and arrive from the area of the storm in the form of swell. For the observer, the external differences between them are reduced to the difference between the actively non-uniformly oscillating surface of the sea and elongated regular shafts in which water particles perform an orbital motion, the radius of which rapidly decreases with depth, tending to zero.

It should be noted that the waves are not at all like currents and tides. The wavelength decreases, the height and steepness increase when wave approaches to the coast. These

\footnotetext{
* Corresponding author: ShilovaLA@mgsu.ru
} 
phenomena are determined by the effect of the bottom (depth of water) [3]. Sea waves are different from radio waves, which move at a constant speed. The greater period of the waves means their greater speed. The energy of sea waves is distributed over a very wide spectrum of wave components, each of which has its own amplitude and period. Each individual wave is generated by the same wind, simultaneously acting over a homogeneous medium, and yet their height may differ in the ratio of $1: 10$. This is explained by the fact that the wave exists for a very short time: not a single storm wave lives more than two minutes. Obviously, the task of identifying waves is very complex; experiments under model conditions on lakes and reservoirs are not able to reproduce the storms of the high seas. The averaged wave power from different sources can be from 40 to100 KW per meter of the wavefront, reaching $150-200 \mathrm{KW} / \mathrm{m}$ at its peak [4].

The main difficulty in the large-scale development of the main non-conventional renewable energy sources is the low energy flux density to be utilized [5]. According to this indicator, the wave energy compares favourably among other renewable energy sources, exceeding the wind energy tens of times in specific indicators [6]. This explains the world's interest in devices that utilize wave energy. However, in terms of the cost of energy received, the efficiency of transforming devices achieved and the scale of development, wave power engineering is significantly inferior to wind energy, where almost $10 \mathrm{GW}$ of equipment capable of more than $\$ 10$ billion is introduced annually [7].

The efficiency of wave power stations is determined by the optimal choice of the water area with a steady supply of wave energy, the station design with devices for smoothing the uneven wave mode. It is believed that wave stations can operate efficiently using a power about $80 \mathrm{~kW} / \mathrm{m}$. Such a power falls every year on every meter of the wavefront from the North Atlantic in Britain. The wave energy reserves can be judged from the following example. A wavelength of one and a half meters and a length of 15 meters carries a power of $4.3 \mathrm{KW}$ : a height of three meters $-17.9 \mathrm{KW}$, a height of six meters - $220 \mathrm{KW}$, a twelve meter - $880 \mathrm{KW}[8]$

\section{Analysis of the strengths and weaknesses of various types of energy converters wave}

All known wave power plants consist of four main parts: the working part; working body; a power converter; fastening systems (Figure 1, a). The working part is in direct contact with water. Under the action of the waves, it makes certain movements or changes the conditions of motion of the waves. The working parts include all kinds of float systems, water wheels and other similar devices. The working body is an environment that acting on the power converter.

The power converter is designed to convert the energy stored by the working part (mechanical energy of the movement of the working part, the differential levels in the pools, air pressure or oil) into electrical energy. Numerous chains, gear and other transmissions, hydraulic pumps and turbines, air turbines, generators, etc. are used as power converters.

The fastening system keeps the wave setup in place. If the installation is floating, it is connected by flexible connections to the vessel transport. If the installation is located on the shore, then the fastening system is the design of the installation.

Usually, in the wave energy converters, two circuits are distinguished: the first circuit perceives the effect of waves on special devices and turns their work into the potential or kinetic energy of the working fluid (most often liquid or gaseous). The second circuit converts the energy of the working fluid into electricity (most often through turbines). Wave power stations differ from traditional ones mainly in the design of the primary circuit. 
The first group of stations uses the principle of "oscillating water column" (Russell rectifier, installation "Kaymer" and other similar structures). Wave power plant "Kaimei" the most powerful station with pneumatic converters built in Japan. It uses a wave height of 6-10 meters. The total capacity of the plant is $1000 \mathrm{~kW}$. The system is simple in design and inexpensive to manufacture, but it has a low efficiency [9]. The second group of stations relates to floating type systems. These are Salter's "diving duck", Cockerel's "contour raft", Parks's "asymmetrical wedge" and other installations. They use complex lever designs but have high efficiency.

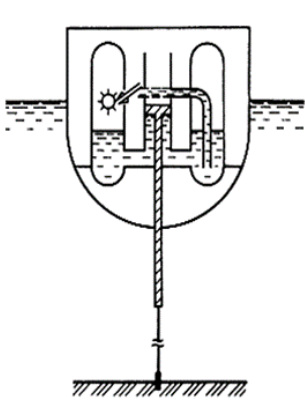

a)

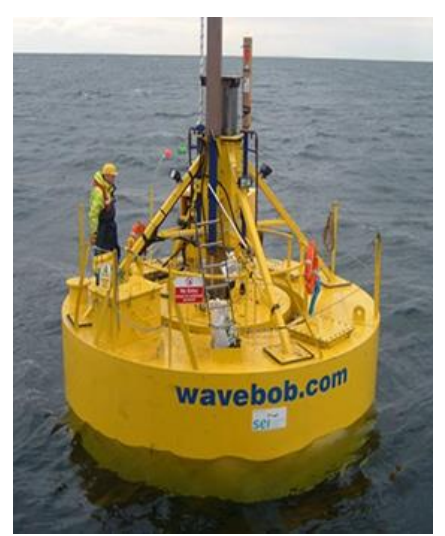

b)

Fig. 1. Wave power plant (schematic diagram) (a) and wave generator WWEC (b). Source: authors' illustration, [10]

Most wave power stations are installed in the open sea at depths of more than 50 meters, since, reaching the coastal waters, the waves lose most of their energy. In this case, one of the main requirements is the stabilization of wave installations and the elimination of the demolition of the stations themselves. For this purpose, supports mounted on the bottom, anchor systems and other types of external and internal stabilization are used. An essential requirement is the orientation of the working bodies relative to the wave travel, damping the movement of an object using special means. It should be noted that the water level is constantly changing. For example, in the North Atlantic, the secular sea level range is 35 meters. An example of an effective solution to this problem is the wave power generator called Wavebob Wave Energy Converter (WWEC) [10,11], designed by the Irish company Wavebob in collaboration with the British and Norwegian companies (Figure 1, b) The installation is a buoy anchored on a long chain, generating energy due to oscillations under the action of waves. Existing float generators are fixed on a chain of a fixed length and this often leads to downtime when the water level changes significantly and the chain is either too short or too long. Irish designers solved this problem. Instead of resting on the waves when the sea level drops or sinks into the water as it rises, WWEC automatically adjusts the length of the anchor chain, always remaining in the optimal position. It is alleged that each unit will produce megawatts of energy.

The advantages of the installation developers include the versatility of the design: WWEC is suitable for seas with any climate. Usually, special versions with a mass slightly larger than for the Mediterranean are made for storm regions.

For many wave systems of float type, it is characteristic to transform the swing of a lever with a float under the action of a wave into rotational movement of the power take-off shaft using various kinds of the ratchet, cable and other mechanisms. The number of such pendulum devices varies over a wide range and can occupy large areas on the surface of the water. However, in this case, it is also necessary to have fixed support, relative to which the floating elements swing. In most cases, columnar structures fixed at the bottom of the sea are used as supports. 
The most famous design of this type is the "Cockerel raft" [12], which is a hinged chain of three pontoons, with sea waves taking the outlines of the sea surface (Figure 2, a)

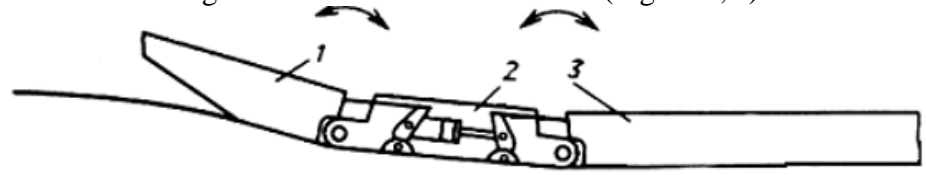

a)

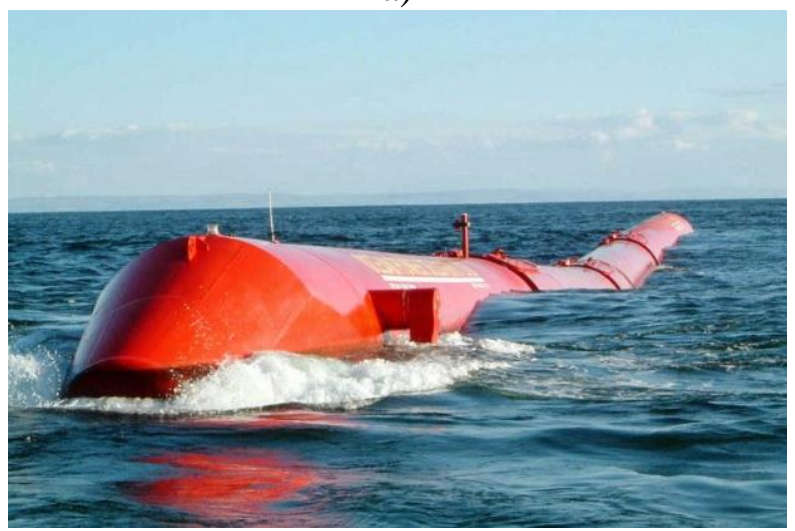

b)

Fig. 2. Cockerel's raft (1, 2, 3 - pontoon number) (a) and full-scale prototype Pelamis P-750 (b). Source: $[12,13]$.

The front pontoon 1 moves freely up and down, obeying oscillations of the waves. The movements of the second pontoon 2 are more limited because the surface of the water beneath it becomes flatter after most of the wave energy is intercepted by the first pontoon. Third pontoon 3 in the chain twice as long as the first two and relatively more stable. Thus, the work of the raft is based on the relative turns of adjacent pontoons. Each hinge through two long connecting rods and special levers connected to the pistons of the hydraulic cylinders. Raft movement causes the pistons to move back and forth, pumping fluid in an isolated closed system. The fluid is pumped through four nozzles and under low pressure flows from the reservoir under the piston, and under high pressure is supplied from the working side of the piston to the pipe and then to the turbine, the shaft of which is connected to the generator shaft. The whole design of the raft is fixed anchors. In the case of very long waves, the energy at such wave power plants is not produced, because then all three pontoons are a single float and the drives in the articulated clutches are fixed.

The raft works more efficiently when its length is close to the wavelength. The maximum efficiency of the raft can be with hundred-meter waves with a height of 10-12 meters. For such constructions, an important task is their anchoring. It should be noted that one of the advantages of such raft is that it allows large waves to roll over themselves. The current model of the raft on a scale of 1:10 produces electricity with a power of $1 \mathrm{~kW}$ from waves whose length is 10 times less than the wavelength of the open sea. A full-scale generator with a length of about 100 meters can provide a stable $2 \mathrm{MW}$. Various hydrostatic systems immersed in water, in which soft shells and membranes are used as working bodies, are not discussed here.

In Portugal, a sample of a wave energy converter was fabricated in the form of semisubmerged structures consisting of four cylindrical sections connected by hinges (Figure 2, 
b). These converters are called Pelamis P-750 [13]. Each of them is the size of a small train: 120 meters long, 3.5 meters in diameter and weighing 750 tons.

Waves force this floating "sea snake" to bend, due to which inside, at the junction points of adjacent sections, hydraulic pistons move, pumping oil through hydraulic motors, in turn, rotating electric generators. The electricity produced by one "snake" is sent to the cable that descends from the float to the bottom. Several such "snakes" can be electrically connected directly at their location. And then by one cable, all the total power will be supplied to the shore. Most of the Pelamis floats are occupied by three Power Conversion Modules with a capacity of 250 kilowatts each. Thus, the power of one converter is 750 kilowatts, and the whole "Portuguese trio" is about 2.25 megawatts.

The project, known as Salter's "duck" [14], is also a wave energy converter (Figure 3, a). This system consists of supporting walls with ballast and a moving element ("duck"), moving around the axis in accordance with sea level fluctuations. Several "ducks" are interconnected by a support shaft, which is rotated with the help of ratchets on each "duck". The continuity of the shaft rotation is ensured by the fact that at least one "duck" of several planted on the shaft tends to turn it in the right direction. After this "duck" ceases to take the effects of the wave, there will always be another one on the rise of any wave.

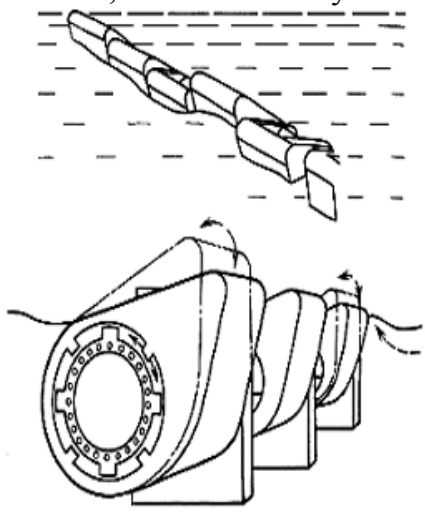

a)

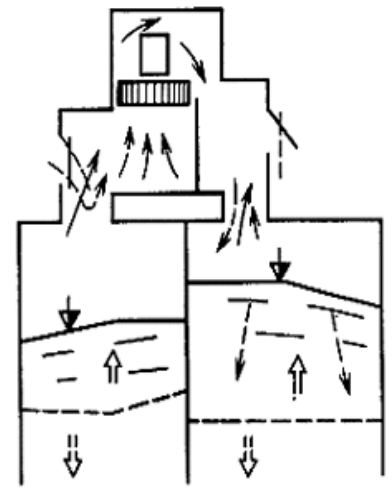

b)

Fig. 3. "Salter's Duck" (a) and Masuda's water column (b). Source: $[14,15]$.

There is another design diving "duck". It also has several "ducks" on one supporting shaft, each of which drives several hydraulic pumps located inside the shaft. The pumps, in turn, supply water under pressure to a hydraulic turbine, on the same shaft with which the generator rotor is located. It is believed that from one meter of the chain consisting of the described "ducks", it can be got an average of 30 to $50 \mathrm{~kW}$ of power, and with a chain of $480 \mathrm{~km}$ long, it can be satisfied all modern electricity needs of whole Great Britain. The diameter of the support shaft of such a chain reaches $15 \mathrm{~m}$. The size of the "duck" is close to the size of a small cottage. The most difficult problem in using the principle of swinging "duck" is the coordination of the movement of "ducks" with constantly changing wave parameters (height, frequency, direction).

Another type of wave power station is Massoud's pulsating water column [15] (Figure 3, b). Structurally, this device is a floating inverted tank, the lower open part of which is submerged under the lowest level of water (troughs of the wave). When raising and lowering the water level in the tank, cyclical compression and expansion of air occur. Air flow through the valve system drives the turbine wheel located in the hole at the top of the tub.

The advantages of the Masuda pillar consist in the absence of significant moving elements in its design, the use of an air turbine with a high rotational speed, and a slight dependence 
of efficiency on the direction of wave movement. However, the efficiency of the Masuda column strongly depends on the frequency of oscillation of the waves, reaching a maximum in the range of $1.2 \ldots 1.3 \mathrm{~Hz}$. The construction of the Massouda pillar was perfected in England, where it was called an oscillating pillar.

\section{The problem of protection of wave installations in extreme weather conditions}

A review of publications on wave energy showed large number of proposals, patents and design solutions, but very little attention is paid to the protection of wave installations from the destructive effects of waves, especially during stormy weather $[16,17]$. The force of the impact of the waves can be judged, for example, by the fact that a breakwater weighing seven thousand tons was overturned in Scotland. It was replaced by a fourteen-ton structure, which was also destroyed. In a heavy American cruiser, a thirty-ton piece of the bow of the vessel was blown off by a wave [9]. In 1985, in Norway, an industrial wave station was built that operates according to the pneumatic principle. It was a reinforced concrete chamber in the rock, under which was installed a steel tower with a height of 12.3 meters and a diameter of 3.6 meters. Annual electricity generation was 1.2 million $\mathrm{kW} / \mathrm{h}$. In the winter storm of 1988 , the station tower was destroyed.

Ensuring the survivability of wave power stations in extreme conditions of storm waves is possible in two versions: free transmission of dangerous waves; immersion of wind power plants under the surface of the water in the deep layers. Oil platforms are firmly mounted on the bottom of the supports freely pass waves, instead of taking them on themselves, thereby preventing the effective damping of the waves. However, this method is not suitable for most wave systems, since reducing the interaction with the wave of a floating element of a wave-receiving system dramatically reduces the power take-off and its conversion efficiency [19].

To confirm this conclusion, let us determine the magnitude of the dynamic effect of wind waves on the floating elements of wave power plants (WPP). The waves incident on the floating structures of the WPP are partially reflected and partially pass under the barrier, flowing around it. In this case, for simplicity, we assume that the plane of the WPP housing is impermeable to liquid particles; the cross section of the floating structure has a rectangular shape, the body is rigid. In this case, the energy of the incident wave must be equal to the sum of the energies of the reflected and transmitted waves. To determine the force effect of waves on a stationary or non-stationary floating object, it suffices to find the difference between the energies of these waves [20].

$$
\Delta \mathrm{F}=\mathrm{F}_{1}-\mathrm{F}_{2}
$$

Where $F_{1}, F_{2}$ - the power of the wave of pressure on the bow and stern surface.

When considering the interaction of waves with floating elements of the WPP design, three cases are possible: $\mathrm{h}>\mathrm{z}, \mathrm{h}<\mathrm{z}$ and $\mathrm{h}=\mathrm{z}$ (where $z$ is the height of the floating object, $h$ is the wave height). If $h \leq z$, in this case the wave load is completely transmitted to the wave absorber. If $\mathrm{h}>\mathrm{z}$ - the nose of the body will be completely buried in the water and in this case it is necessary to take into account the additional load from the flooding of the nose.

Define $F_{1}$ for the case $h \leq z$. The excess hydrodynamic pressure at the point of the wave at the depth $z$ is determined by the formula written for the coordinate system shown in the calculation scheme (Fig. 4): 


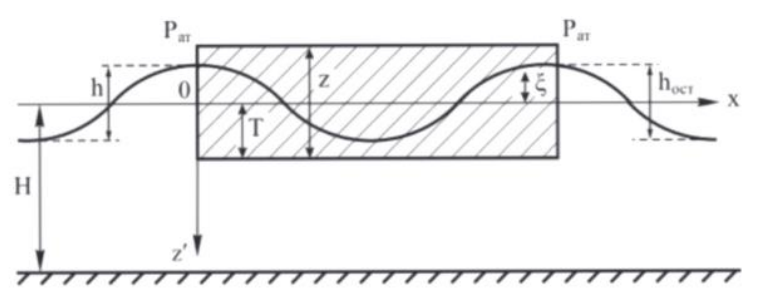

Fig. 4. The scheme for calculating the determination of the dynamic effect of wind waves on the floating elements (hull) of a wind farm.

$$
\frac{\mathrm{P}-\mathrm{P}_{\mathrm{at}}}{\rho}-\mathrm{gz}-\mathrm{g} \frac{\mathrm{ch}[\mathrm{k}(\mathrm{H}-\mathrm{z})]}{\mathrm{ch}(\mathrm{kH})} \xi
$$

where $\xi$ is the ordinate of the wave profile points; $P$ - hydrodynamic pressure at the point of the wave; Pat is a pressure on the free surface of the liquid; $g$ is a gravitational acceleration; $k$ is the wave number $(\mathrm{k}=2 \pi / \lambda) ; \lambda$ is the wavelength; $\rho$ is the density of water.

For the received coordinate system in a plane coinciding with the front and rear face of the housing, the wave profile $(\zeta)$ at the front $\left(\zeta_{1}\right)$ and the rear face $\left(\zeta_{2}\right)$ is described by two equations:

$$
\zeta_{1}=\frac{\mathrm{h}}{2} \cos \sigma \tau \quad \zeta_{2}=\frac{\mathrm{h}_{\mathrm{ocr}}}{2} \cos \sigma \tau
$$

where $\sigma=2 \pi / \tau$ - the angular velocity or the angular frequency; $\tau$ is the wave period.

The pressure force on the front and rear $\left(\mathrm{F}_{1,2}\right)$ face with area $\mathrm{S}$ is determined by integrating the expression:

$$
\mathrm{F}_{1,2}=\mathrm{g} \rho \int_{\mathrm{S}}\left(\mathrm{z}-\frac{\mathrm{ch}[\mathrm{k}(\mathrm{H}-\mathrm{z})]_{1,2}}{\operatorname{ch}(\mathrm{kH})} \xi_{1,} \mathrm{~S}\right.
$$

$\mathrm{dS}=\mathrm{Bdz}$ (where B is the width of the WPP case, $\mathrm{dz}$ is the elemental height).

The magnitude of the pressure force on the front face can be determined by integrating (4) within the limits of the draft of the WPP case $\mathrm{T}$ to $\mathrm{T}+\xi$. After the integration, we obtain:

$$
\mathrm{F}_{1,2}=\frac{1}{2} \rho g B\left(2 \mathrm{~T} \xi_{1,2}+\xi_{1,2}\right) \frac{\rho g B \xi_{1,2}}{\mathrm{k} \cdot \operatorname{ch}(\mathrm{kH})} \cdot\left[\operatorname{sh}\left[-\mathrm{k}\left(\mathrm{H}-\mathrm{T}-\xi_{1,2}\right)\right]-\operatorname{sh}[-\mathrm{k}(\mathrm{H}-\mathrm{T})]\right]
$$

The authors' studies show that the change in wave height at the front face of the body due to its partial reflection is insignificant and is less than $0.5 \%$. that, for practical calculations, can be ignored [21].

Wave damping can be estimated by the coefficient $\beta=$ host/ h, the value of which is taken from 0 to 1 (with $\beta=0$, there is a complete damping of the wave, and for $\beta \sim 1-$ the damping of the wave does not occur).

Thus, in determining the pressure force on the food casing surface WPP be administered in the calculation value of the residual value of the wave height host $=\beta \cdot h$, the calculation scheme shown in (Fig. 4).

Having determined the force of wave pressure on the bow and stern surfaces, using (1) one can find the magnitude of the force effect on the WPP.

The obtained calculated values of the pressure force of a wave of $30 \mathrm{~m}$ in length on a WPP design per 1 running meter of a floating object, calculated using this technique, are presented in Table 1. From the calculation formula (5), it follows that in the case of weak wave damping $(\beta \sim 1)$ the value of the pressure force of the wave on the structural 
elements of the WPP will be close to zero, which in turn dramatically reduces the efficiency of power take-off and the conversion of wave energy (Table 1).

Table 1. The calculated values of the wave pressure force $(\lambda=30 \mathrm{~m})$ on the WPP design and coefficient $\beta$.

\begin{tabular}{|c|c|c|c|c|}
\hline $\mathbf{h}, \mathbf{m}$ & $\mathbf{1}$ & $\mathbf{2}$ & $\mathbf{3}$ & $\mathbf{4}$ \\
\hline $\boldsymbol{\Delta} \mathbf{F} \mathbf{k N}$ & 5 & 30 & 50 & $90 \mathrm{kN}$ \\
\hline $\boldsymbol{\beta}$ & 0.95 & 0.62 & 0.41 & 0.18 \\
\hline
\end{tabular}

The averaged parameters of WWEC $[10 ; 11]$ were used in the performed calculations as the initial data characterizing the design and operation mode of the WPP (draft $=\mathrm{T}=1.2 \mathrm{~m}$; width $\mathrm{B}=8 \mathrm{~m}$; depth $\mathrm{H}=10 \mathrm{~m}$ and wavelength $\lambda=30 \mathrm{~m}$ ).

It is obvious that it is preferable to protect the system of wave installations of float type by submerging them underwater at a certain depth during the dangerous effects of a storm. In devices using anchor systems, such immersion can be achieved by reducing the length of the anchor chain. Naturally, this will require the development of the necessary mechanisms and control devices for them, which will significantly complicate the design of the wave energy system and its maintenance.

In all cases of the use of wave systems in the open sea, there is also a problem of transferring electricity to the coast via cables, which must resist the marine environment, to withstand heavy traffic at attachment points to objects located at sea. No less important is the problem of survival in the open sea designs of wave generators for floating systems in the form of a "raft".

Of the variety of methods for converting wave energy into electrical energy, most represent systems that use the energy of sea waves on their surface. The movement of the rolling element to convert the energy of the waves of such systems relative to the stabilized part of the object occurs in the following movement variants: vertical oscillations, horizontal oscillations, angular oscillations, rotational and volume movements.

Ways to stabilize wave energy systems can be represented as follows. External stabilization: anchor system, rigid bottom supports, coastal structures. Oscillatory stabilization: system oscillations in a liquid medium with a phase shift relative to the oscillations of the medium itself, deepening of the vibrational part of an object into unperturbed layers of a liquid medium. Braking stabilization: breaking of a part of the system using screens embedded in undisturbed layers of a liquid medium. Phase stabilization: the spatial arrangement of several working bodies of an object at points with different phases of medium oscillation along the wavefront, the spatial arrangement of several working bodies of an object at points with the opposite phase of medium oscillations along the wave beam [8]. The specified types of stabilization are focused on the operation of the wind farm for a specified time.

\section{Conclusions}

The most acceptable type of protection against storm impact is the method of penetrating the vibrational part of an object into unperturbed layers of a liquid medium. In this embodiment, it is preferable to use external stabilization in the form of rigid bottom supports (columnar structures) and anchor systems. In all cases, during a storm, special devices are necessary to immerse floating elements of a wind farm to a certain depth and hold them there during a storm. When reducing to a predetermined level of storm exposure, the entire system should automatically return to its original position. To activate the mechanisms and devices for immersing floating elements of a WPP, an independent source of energy is needed, since, while immersed in the working elements, they will not be able to 
fully provide the drive with electric power for immersion, holding and returning (ascent) to the initial position.

Renowned sources of marine-based energy are systems that combine the conversion of the energy of sea waves and wind into electrical energy. Such structures, being autonomous systems, work on one load, complementing each other. Obviously, the use of wind turbines installed above the level of the waves on the same base with a wave power plant (for example, on a single column support fixed at the bottom) is advisable to use as a source of mechanical or electrical energy driving the mechanisms for deepening and holding during the storm undisturbed water layers. It is possible to use hydraulic devices that pump oil into the tight cavities of the immersion mechanism. Such devices should have valves that are automatically triggered during a gale wind perceived by a rotary wind turbine with a vertical shaft. Such wind turbines do not require feathering, do not depend on the wind direction and are simple in design. Other systems are not excluded: electrical, electromechanical, pneumatic, etc. for use as immersion devices of a wave station during a storm. To power these devices, it is advisable to use an autonomous engine that automatically connects the dive system at a dangerous level of storm exposure. It is possible to use storm protection of wind power stations with their anchor stabilization, using an independent source of energy to change the length of the anchor chains for immersion

This topic was funded by the state assignment, theme No. 0149-2019-0002.

\section{References}

1. N. Khan, A. Kalair, N. Abas, A. Haider RSER 72, 590-604 (2017)

2. A. Muetze, J. G. Vining Ocean Wave Energy Conversion Energy 00 1410-7 (2006)

3. M. McCormick 1985 Wave energy transformation (Moscow, 1985)

4. V. I. Sichkarev, V. A. Akulinichev, Wave energy stations in the ocean (Moscow: Science, 1989)

5. A. A. Solovyov RSE 217-26 (2014)

6. D. A. Solovyov The energy of the hydrosphere (Moscow, PH "Energy", 2011)

7. K. S. Degtyarev, A. M. Zalikhanov, A.A. Soloviev, D. A. Soloviev ETE 10-20 (2016)

8. V. Sichkarev The Basics of Research and Development of Wave Power Stations (Vladivostok, Far Eastern Scientific Center, USSR Academy of Sciences, 1987)

9. D. Ross Wave Energy (Leningrad, 1981)

10. Electric generator that uses the energy of sea waves - Science and technology - Energy - Compulenta Compulenta https://archive.li/wJ0RR

11. F. Mouwen Presentation to Engineers Ireland 2008 Eng. Ireland. (2016)

12. Chapter 7. Ship with broken http://www.plam.ru/tehnauka/yenergija_voln/p11.php

13. New Wave Power Plant

http://www.ecotoc.ru/alternative_energy/gidroenergetik/d176/

14. Principle of the Solter Duck. Oscillating water column of wave energy http://geyz.ru/load/ehnergija_voln/upravlenie_ehnergiej_voln/princip_solterovskoj_utk i_oscillirujushhij_vodnyj_stolb_ehnergii_volny/11-1-0-95

15. Oscillating Water Pole http://msd.com.ua/vozobnovlyaemye-i-vtorichnye-istochnikienergii/koleblyushhijsya-vodyanoj-stolb

16. A. A. Solovyov, D. A. Solovyov PIG 4, 45-52 (2017)

17. C. Frid, E. Andonegi, J. Depestele, A. Judd, D. Rihan, S. I. Rogers, E. Kenchington IAR 32, 133-139 (2012) 
18. Anon Oil Platform in a Strong Storm http://earth-chronicles.ru/news/2015-01-1475338

19. V.G. Chigirenko Waves in deep water and shallow water (Moscow: Laboratory of Hydrodynamics, Institute of Mechanics, Moscow State University "Sputnik", 2005)

20. V. P. Korpachev Theoretical basis of water transport of wood (Krasnoyarsk6 2009)

21. A. M. Basin, V. M. Anfimov Vessel hydrodynamics (Ship hydrodynamics, Leningrad, 1961) 